\title{
Improving Understandability of Declare Models by Revealing Hidden Dependencies
}

\author{
Johannes De Smedt, Jochen De Weerdt, Estefanía Serral, and Jan Vanthienen \\ KU Leuven Faculty of Economics and Business \\ Department of Decision Sciences and Information Management \\ Naamsestraat 69 \\ B-3000 Leuven, Belgium \\ firstname. lastname@kuleuven. be
}

\begin{abstract}
Declarative process models have become a mature alternative to procedural ones. Instead of focusing on what has to happen, they rather follow an outside-in approach based on a rule base containing different types of constraints. The models are well-capable of representing flexible behavior, as everything that is not allowed by the constraints in the model is possible during execution. These models, however, are more difficult to comprehend and require a higher mental effort of both the modeler and the reader. Since constraints can be added freely to the model, it is often overseen what impact the combination of them has. This is often referred to as hidden dependencies. This paper proposes a methodology to make these dependencies explicit for the declarative process modeling language Declare by considering a Declare model as a graph and relying on the constraints' characteristics. Moreover, this paper also contributes by empirically confirming that a tool that can visualize hidden dependency information on top of a Declare model has a significant positive impact on the understandability of Declare models.
\end{abstract}

Keywords. Declarative process modeling, Declare, Hidden dependencies, Empirical evaluation

\section{Introduction}

Declarative process models have been proposed to counter the flexibility limitations of procedural modeling languages. Instead of modeling predetermined paths of activities, declarative process models use constraints to express what can, cannot, and must happen. Every execution sequence that is not strictly forbidden by the constraints can be enacted by the model. This makes declarative models much more flexible, but also more difficult to comprehend. To put it simplistically, it is not possible to 'find an execution path by following your finger along the arcs'. There are many possible outcomes due to the interaction of the constraints over the activities.

\footnotetext{
* Corresponding author.
} 
In different works approaches to deal with the understandability problems of declarative models have been proposed. For instance in [1], the impact of hierarchy is investigated and in 2] the typical pitfalls of understanding declarative models are pointed out.

This paper proposes an approach capable of improving the understandability of models expressed in Declare [3, one of the most widely used declarative process modeling language. The approach deals with hidden dependencies 22415, one of the main reasons that make Declare models difficult to understand. Hidden dependencies pose a significant challenge for humans: it is not sufficient to rely on the information explicitly indicated by the constraints, but one has to carefully analyze all the defined constraints for understanding all the dependencies that are not explicitly visible (i.e., that are hidden). The contribution of this paper consists of a methodology to build so-called dependency structures in order to reveal all hidden dependencies and make them explicit in a Declare model. Furthermore, this methodology is developed into the Declare Execution Environment 1 , a tool that supplements an existing Declare model with visual and textual annotations to clarify to the user which behavior is allowed or disallowed by the model. In an experimental evaluation with 95 novice Declare modelers, we show that the methodology to make hidden dependencies explicit and visually annotating a Declare model with this information, has a significant positive impact on the understandability of Declare models.

The structure of the paper is as follows. First, the concept of Declare constraints is briefly summarized and relevant characteristics are explained. Next, Section 3 explains how to capture and formalize dependency structures, followed by Section 4, which shows the implementation and tool. This tool is used for experimental validation in Section 5. Finally, Section 6 summarizes the related work and Section 7 discusses future work and the conclusion.

\section{Declare Constraints and Their Characteristics}

Declare models are constructed using a fixed set of constraints, which are summarized in Table A1 in [6. They range from unary constraints, indicating the position and cardinality of an activity, to $n$-ary constraints, which capture typical sequence behavior such as precedence and succession relationships. A Declare model $D M=(A, \Pi)$ can be represented as follows:

- $A$ is a set of activities from the alphabet $\Sigma$,

- $\Pi$ is the set of Declare constraints defined over the activities.

In this paper we assume $n \leq 2$. A Declare graph can be represented as a directed graph $D G=(A, \Pi)$. Hence the activities and constraints map one-to-one onto the graph in case of $n=2$, given that unary constraints are considered self-loops. We denote all incoming arcs of $a \in A$ as $\bullet \subseteq \subseteq \Pi$ and outgoing arcs as $a \bullet \subseteq \Pi$. The antecedent and consequent of $\pi \in \Pi$ are denoted as $\pi_{a}$ and $\pi_{b}$.

\footnotetext{
1 http://www . processmining.be/declareexecutionenvironment
} 
The execution of a Declare model can be realized by constructing an automaton (either a Büchi 7] or finite state automaton [8] ) by multiplying the different constraints' automata to obtain the behavior that is allowed for by all of them. This multiplication actually abolishes the notion of the separate constraints and thus throws away the information of how the separate constraints interact. One technique to mitigate this is to color the constraints 10 by keeping both the global and separate automata, but still the interactions are untraceable.

Declare constraints exhibit a hierarchy, which is well-explained in [7/8]. For unary constraints, Existence $(A, n)$ and $\operatorname{Absence}(A, n)$ together form $\operatorname{Exactly}(A, n)$. Binary constraints are divided in different classes, for which every class depends on the previous one: Unordered (Responded/co-existence), Simple ordered (Precedence (p), Response (r), Succession (s)), Alternating ordered (Alternate $p, r, s$ ), and Chain Ordered (Chain $p, r, s)$. Next to these constraints, there exist negative versions for three of them (Not co-existence, Not succession, Not chain succession). Finally, the Choice constraint exists, which is comparable with a branched unary constraint Existence $(\{A, B\}, n)$.

For binary constraints, (Alternate/Chain) Response(A,B) and (Alternate/ Chain) Precedence $(A, B)$ form (Alternate/Chain) Succession. When a property is discussed for, e.g., Chain succession, this hence also includes (Chain/Alternate) precedence/response and vice versa.

Furthermore, each constraint has specific characteristics that are discussed in [11. Some constraints have an impact on the temporary violation aspect of the model (the constraints is not in an accepting state and requires an activity to resolve it, e.g. Response or Choice), some constraints can disable activities for the remainder of the execution (such as Exactly and Not succession), and some constraints can temporarily block all other activities (Chain constraints). These different characteristics all impose a certain dependency among constraints that is not directly visible through a single constraint (arc). E.g., a model consisting of $A=\{a, b, c\}$ and $\Pi=\{\operatorname{Response}(a, b), \operatorname{Response}(b, c), \operatorname{Exactly}(c, 2)\}$ contains a hidden dependency between $a$ and $c$. When $c$ is fired once (and hence can only fire one time anymore), and $a$ has fired without $b$ firing already, $c$ should not fire before $b$ resolves the temporary violation of $\operatorname{Response}(a, b)$, since after firing $c$, $c$ cannot resolve Response $(b, c)$ anymore (as it can only fire two times) and $b$ should not fire to avoid another temporary violation of Response $(b, c)$.

\section{Declare Dependency Structures}

This section discusses how dependency structures retrieved from Declare models can be constructed (Section 3.1), how they can aid interpretation of the model and the way in which constraints interact (Section 3.3). Before constructing the structures, however, the unary constraints in the model need to be propagated to achieve the correct interpretation, as explain in Section 3.2 


\subsection{Construction}

The characteristics explained in Section 2 are now used to assess the impact of every constraint on the rest of the model.

A hidden dependency is defined as an interaction between constraints and their activities that is not made explicit as such in the model itself. They are the outcome of multiplying the separate constraints to avoid permanent violation, as explained earlier. Hence, it is paramount to find the ways to avoid permanent violation to occur. There are three types of resolution strategies to resolve temporary violations:

1 An activity still must happen: after firing the antecedent in Responded existence, Co-Existence, (Alternate/Chain) Response, the consequent must fire afterwards.

2 An activity still must happen a certain amount of times: Existence, Exactly, Choice.

3 An activity still must happen at a fixed moment in time: Chain response.

Note that combining different constraints could lead to coalesced resolution strategies: Chain response $(a, b)$ coupled with Existence $(a, 2)$ requires firing $b$ at least twice on certain fixed moments (directly after $a$ ) as well.

Now we construct the set of dependency structures $D P$ for $D M$ with $D S=$ $\left(\pi^{D S}, \Pi_{d e p}^{D S}, D S_{d e p}^{D S}\right), D S \in D P$ with

$-\pi^{D S}$ the constraint triggering the structure,

$-\Pi_{d e p}^{D S}$ the set of dependent constraints, and

- DS dep the set of nested dependency structures dependent of $\pi$.

To fill $\Pi_{d e p}^{D S}$ and $D S_{d e p}^{D S}$, Algorithm 1 creates a dependency structure for every activity that is involved in at least one of the five constraints that can permanently disable it. Hence, a structure is created for $a$ in Absence/Exactly(a,n), a and $b$ in Exclusive choice/Not co-existence( $a, b)$, and for $b$ in Not succession $(a, b)$ as can be seen in Algorithm 1, lines 7-25.

First, all backward-propagating constraints are considered $\left(\Pi_{B W} \subseteq \Pi\right.$, inferred from resolution strategy 1 and used for recursive search, as well as stored in $\Pi_{d e p}$ (Algorithm 2, lines 1-22). During this procedure, all incoming Existence and Choice constraints (as in 2 are stored as well (Algorithm 2, lines 16-18). They also need to be fulfilled, but do not propagate due to their unary nature. When Responded existence is encountered, a new dependency structure $D L \in D S_{d e p}^{D S}$ is constructed because when the constraint becomes satisfied (by firing its consequent), it is satisfied indefinitely (unlike, e.g., Response which can become temporarily violated again) and its propagation is also abolished (Algorithm 2, lines 6-10).

For every activity that is encountered by the algorithm, a forward-dependency search is performed for all forward-propagating constraints $\Pi_{F W} \subseteq \Pi$, which includes all (Alternate/Chain) precedence constraints and Co-existence. These 
constraints need to be activated (the antecedent has to be fired, in the case of alternating versions even multiple times) to resolve dependencies from backwardpropagating constraints. The constraints dependent of them are linked to them through a separate, nested dependency structure $D L \in D S_{\text {dep }}^{D S}$ (Algorithm 2 , lines 22-36).

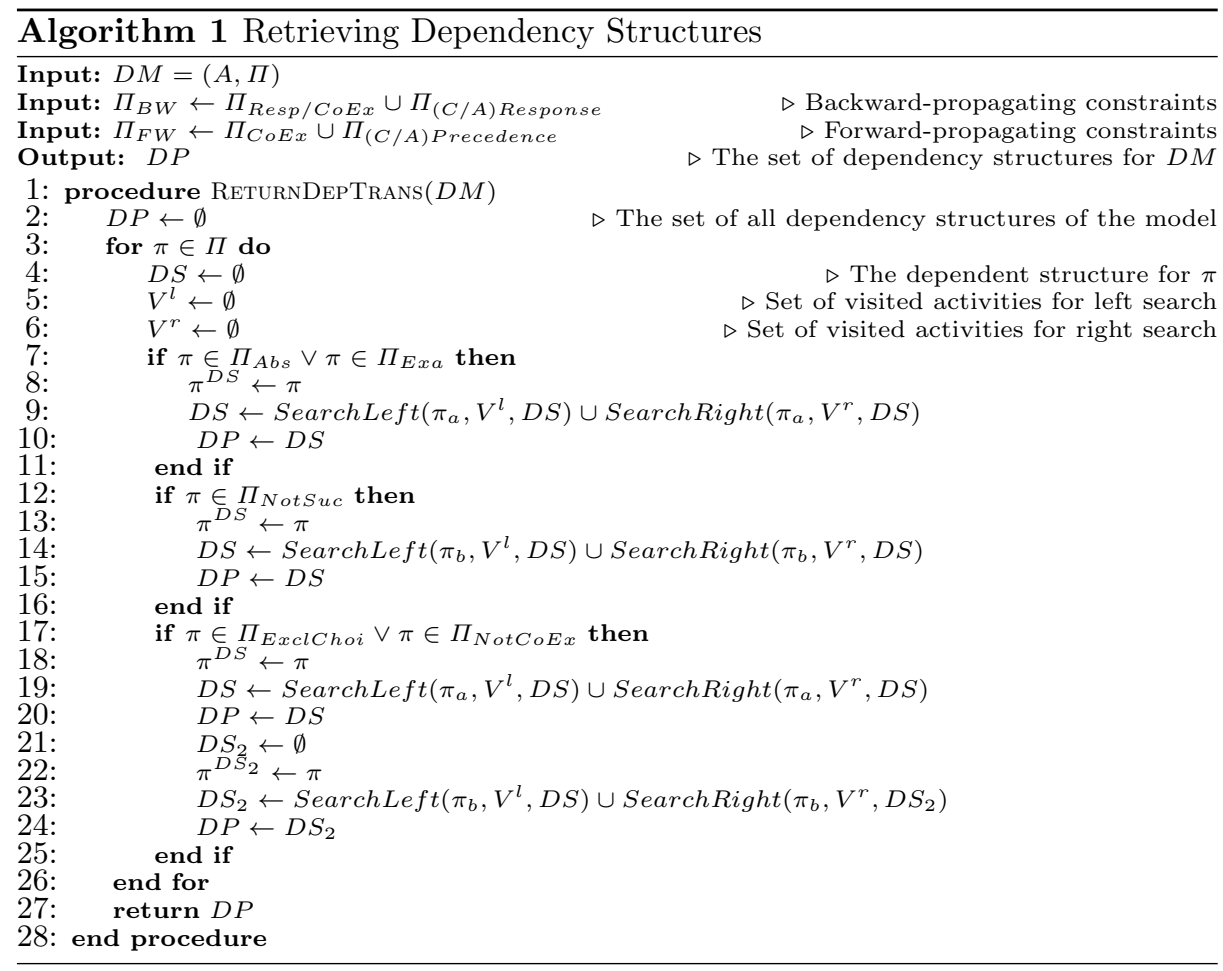

Example Consider the model in Figure 1a. Not succession $(c, b)$, so any occurrence of c cannot be followed eventually by $b$, causes the algorithm to construct a dependency structure for $b$. Backward-searching will reveal Response $(a, b)$ and $\operatorname{Exactly}(a, 1)$ as dependent constraints. $c$ cannot fire before $a$ has resolved $E x$ $\operatorname{actly}(a, 1)$, which will render Response $(a, b)$ temporarily violated and requires $b$ to resolve it. Hence sequences such as $\sigma=e \rightarrow b$ or $\sigma=a \rightarrow e$ are not possible. In a forward search, Precedence $(b, d)$ requires a new dependency structure,

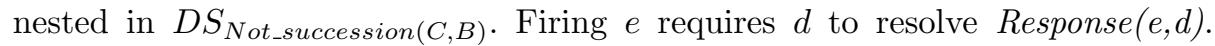
Hence, firing $c$ before firing $e$ would render $e$ disabled, as $b$ can never fire anymore due to Not succession, hence the Precedence $(b, d)$ can never be activated. Firing $b$ before $c$ would resolve this, as $d$ can then fire an unlimited amount of times. The full dependency structure present in the model is $D S=\{\pi=$ 


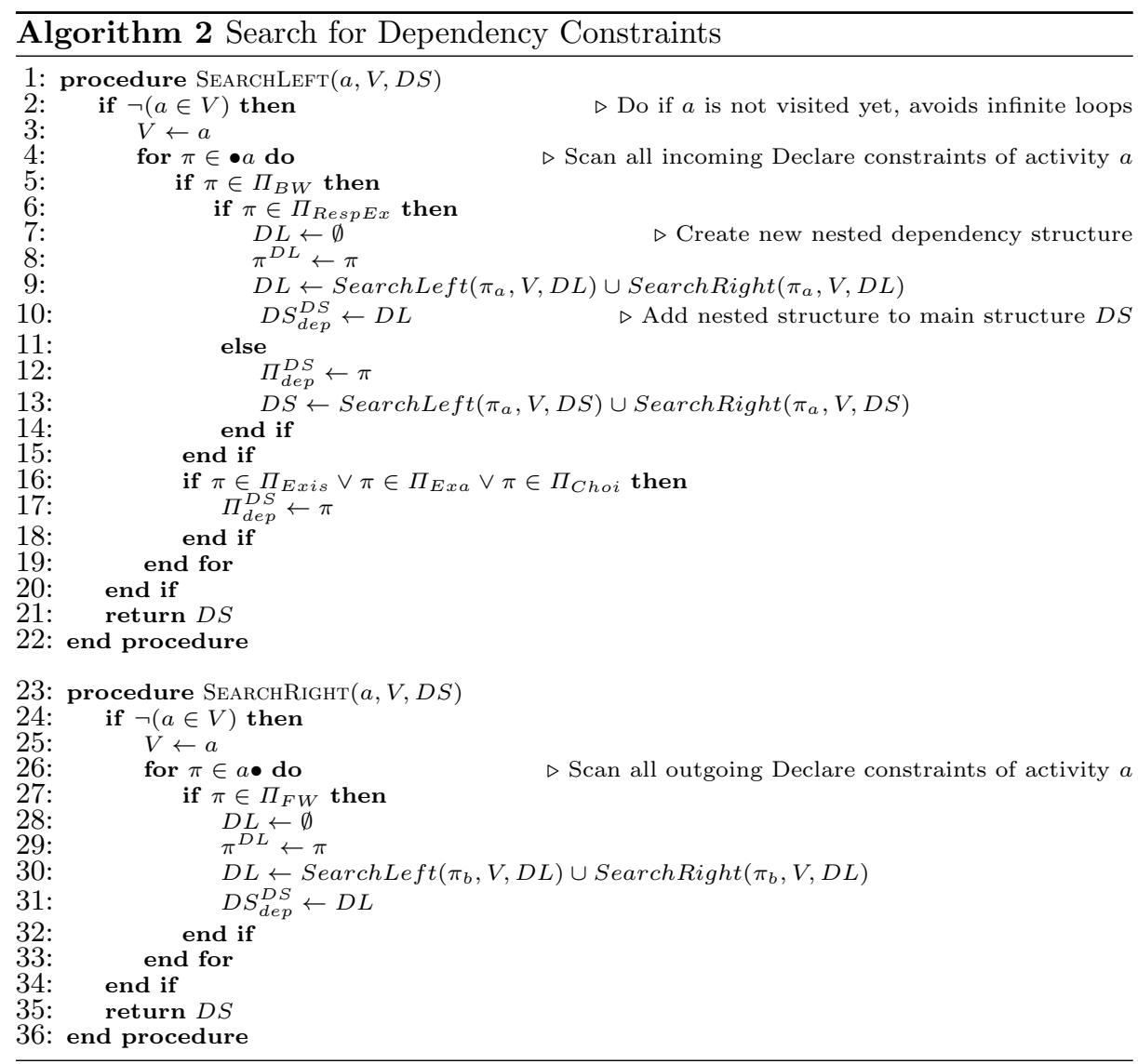

Not_succesion $(c, b), \Pi_{\text {dep }}=\{\operatorname{Response}(a, b), \operatorname{Exactly}(a, 1)\}, D S_{d e p}=\{\pi=$ Precedence $\left.\left.(b, d), \Pi_{\text {dep }}=\operatorname{Response}(e, d), \emptyset\right\}\right\}$.

\subsection{Unary Propagation}

The construction and use of dependency structures depends on the correct propagation of all unary relations inside of the model. E.g., consider the model in Figure $1 \mathrm{~b}$. Changing Precedence $(b, d)$ and Response $(e, d)$ to their alternating version and adding Existence (e,2) would require $d$ and hence $b$ to fire at least twice as well. In general, unary constraints that are not present in the original model are added in the following fashion:

- Responded existence $(a, b)$, if $a$ occurs at least or exactly $n$ times, then $b$ should occur at least once.

- Co-existence $(a, b)$, if $a(b)$ ccurs at least or exactly $n$ times, then $b(a)$ occurs at least once. 


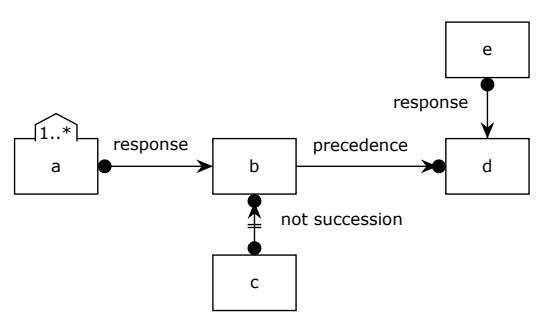

(a) Simple example with Not succession inflicting hidden dependencies.

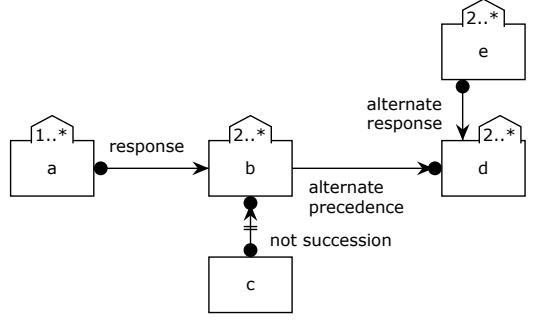

(b) The same example with unary and alternating constraints.

Fig. 1: An example of a small Declare model with hidden dependencies in two variants.

- Response $(a, b)$, if $a$ occurs at least or exactly $n$ times, then $b$ should occur at least once.

- Precedence $(a, b)$, if $b$ occurs at least or exactly $n$ times, then $a$ occurs at least once.

- Succession( $a, b)$, if $a$ or $b$ occur at least or exactly $n$ times, then the other activity occurs at least once.

- Alternate response $(a, b)$, if $b$ occurs at most or exactly $n$ times, then $a$ occurs at most $n$ times. If $a$ occurs at least or exactly $m$ times, then $b$ should occur at least $m$ times.

- Alternate precedence $(a, n)$, if $b$ occurs at least or exactly $n$ times, then $a$ occurs at least $n$ times.

- Alternate succession( $(a, b)$, both $a$ and $b$ should have the same unary restrictions.

- Chain response $(a, b)$, if $a$ occurs at least or exactly $n$ times, then $b$ occurs at least $n$ times. If $b$ occurs at most or exactly $m$ times, then $a$ can only occur at most $m$ times.

- Chain precedence $(a, b)$, if $a$ occurs at most or exactly $n$ times, then $b$ occurs at most $n$ times.

- Chain succession( $a, b)$, both $a$ and $b$ should have the same unary restrictions.

Every unary constraint has a lower bound Existence(n) and upper bound Absence $(m)$, and they are combined and replaced by an Exactly constraint when $n=m-1$. These rules are applied to the model until no unary constraint changes anymore. If there would be an activity for $n>m-1$, this would mean the model would end up in a permanently violated state.

This propagation is done before the model is used in the algorithms in order to have consistent dependency structures. Next, the same procedure is used to calculate for each activity how many times it still has to execute for every execution step. This helps the dependency structures recognize whether a certain nested structure can be cast off because it can fire a sufficient amount of times. 
Example Returning to the example, Existence $(e, 2)$ is propagated to $d$, yielding an Existence (d,2) and next to $b$ yielding Existence $(b, 2)$. The minimum amount of occurrences is also calculated and updated throughout the execution per activity. This way, the dependency structures will incorporate the unary constraints into the model and indicate that $c$ cannot fire before $b$ has fired its appropriate amount of times to enable $d$ and $e$ to fire at least twice. Initially, $b, d$, and $e$ must execute a minimum of 2 times. $b$ can be disabled after $d$ and $e$ have fired at least once, and $b$ has fired at least two times (once before $d$ fired and once after $d$ fired to grant $d$ another execution because of Alternate precedence $(b, d)$ ), for example sequence $\sigma_{1}=b \rightarrow e \rightarrow d \rightarrow b \rightarrow c$ or $\sigma_{2}=e \rightarrow b \rightarrow d \rightarrow b \rightarrow c$. After this execution, $d$ cannot fire until $e$ is fired because they can both fire only once anymore ( $d$ because of Alternate precedence $(b, d)$ and hence $e$ through Alternate response $(e, d))$ and $d$ has to be able to resolve Alternate response $(e, d)$, e.g. $\sigma_{1} \rightarrow e \rightarrow d$ and not $\sigma_{1} \rightarrow d \rightarrow e$.

\subsection{Interpretation}

Constructing dependency structures can already give extra information by displaying them in a graph showing which constraints interact with the main constraint $\left(\pi^{D S}\right)$ in the structure. However, they can be expressed in extra descriptions to annotate the model in order to help understand why constraints are related and what combined impact they have.

First of all, for Exclusive choice ( $a, b)$ and Not co-existence $(a, b)$, the structures reflect that whenever an activity from either structure is fired (either the one for $a$ or $b$ ), the activities in the other structure become disabled permanently. Indeed, firing any activity in the dependency structure of $a$ or $b$ requires them to fire, hence activating Exclusive choice or Not co-existence. If the structures of $a$ and $b$ share activities, this means the net is not deadlock-free.

Secondly, for Not succession $(a, b), a$ becomes disabled whenever a constraint $\pi \in \Pi_{\text {dep }}^{D S}$ is temporarily violated and needs $b$ to resolve it. Also, dependent structures in $d \in D S_{d e p}^{D S}$ cannot contain any violations in their $\Pi_{d e p}^{d}$ unless the antecedent of the main constraint $\pi^{d} \in D S_{d e p}^{d}$ is activated and can execute a minimum number of times required (as explained in Section 3.2).

For unary constraints, $A b s e n c e(A, n)$ and $\operatorname{Exactly}(A, n)$, this applies as well, with the exception that $a$ becomes disabled when a constraint relies upon it to become satisfied again.

Finally, every execution of activities in Chain constraints should be checked. For each of them, it is checked whether the consequent is available to fire for Chain response, or is the only one available for Not chain succession in order to avoid deadlock.

\section{Tool Support}

The construction of the dependency structures has been implemented in a Declare execution environment, of which the implementation can be found by following the link in the introduction. The tool can read a Declare model saved 
from Declare Designer [12, which, during execution, is supported by descriptions for the hidden dependencies. A screenshot and an example can be found in Figure 2 ,

Furthermore, the dependency structures can be visualized next to the model as a directed graph as well. Finally, the trace created over the model by the user is displayed below the model, aiding the user in understanding the history of the current situation displayed over the model.

The execution semantics are provided by dk.brics.automaton [13] and consists of the product of the separate Declare automata expressed in regular expression, as can be found in [8] and [9].

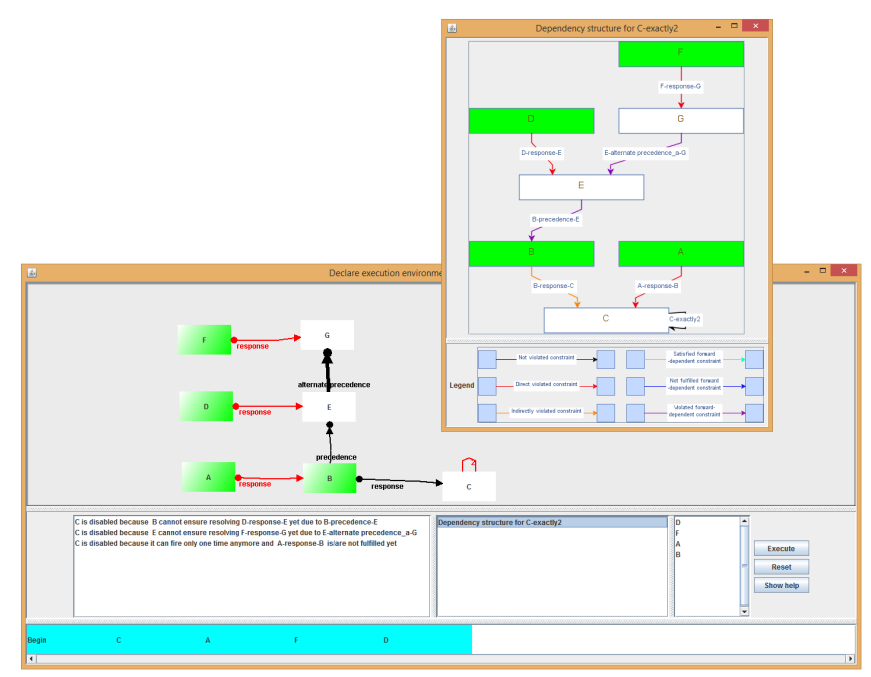

Fig. 2: An example of a small Declare model with hidden dependencies and the corresponding dependency graph for $\operatorname{Exactly}(c, 2)$.

\section{Empirical Evaluation}

Making hidden dependencies explicit by annotating Declare models should significantly improve their understandability. In this section, it is empirically demonstrated that novice process modelers are indeed capable to understand Declare models better when they are provided with an environment that makes hidden dependencies explicit.

\subsection{Experimental Setup}

In our experiment, 95 students (see Table 2 enrolled in KU Leuven's Business Analysis course, in which they learn about both procedural and declarative pro- 
cess modeling, were asked to solve five questions for each of five different Declare models in a timespan of two hours. The students have the same modeling experience and background and can be considered novice business process modelers. The models, as represented in Table 1, are of increasing complexity and are tailored towards assessing different kinds of dependencies:

- Model 1: focuses on the impact of the Not co-existence constraint.

- Model 2: focuses on the impact of unary constraint propagation.

- Model 3: focuses on the impact of simple forward and backward dependencies induced by Exactly $(c, 2)$.

- Model 4: focuses on the impact of more advanced forward and backward dependencies induced by Not succession $(b, e)$.

- Model 5: focuses on the impact of model 3 and 4, with an added Choice constraint.

\begin{tabular}{|c|c|c|}
\hline Model 1 & Model 2 & Model 3 \\
\hline Response(a,b) & Exactly $(\mathrm{a}, 2)$ & Response(a,b) \\
\hline Precedence(b,c) & Existence $(c, 2)$ & Response(b,c) \\
\hline Not co-existence(b,e) & Exactly $(b, 2)$ & $\operatorname{Exactly}(\mathrm{c}, 2)$ \\
\hline $\operatorname{Response}(\mathrm{d}, \mathrm{e})$ & Absence $(\mathrm{d}, 3)$ & Precedence(b,e) \\
\hline & Alternate precedence $(\mathrm{a}, \mathrm{c})$ & Response(d,e) \\
\hline & Alternate response $(\mathrm{b}, \mathrm{d})$ & Alternate precedence $(\mathrm{e}, \mathrm{g})$ \\
\hline Model 4 & \multicolumn{2}{|l|}{ Model 5} \\
\hline Response(a,b) & Response(a,b) & Choice $(a, j)$ \\
\hline Existence(b,1) & Response(b,c) & Not succession $(\mathrm{i}, \mathrm{j})$ \\
\hline Alternate precedence $(\mathrm{b}, \mathrm{c})$ & Exactly $(\mathrm{c}, 2)$ & \\
\hline Not succession $(\mathrm{b}, \mathrm{e})$ & Precedence $(\mathrm{b}, \mathrm{e})$ & \\
\hline Existence $(\mathrm{c}, 1)$ & $\operatorname{Response}(\mathrm{d}, \mathrm{e})$ & \\
\hline $\operatorname{Response}(\mathrm{d}, \mathrm{c})$ & Alternate precedence $(e, g)$ & \\
\hline $\operatorname{Existence}(\mathrm{d}, 2)$ & Response(f,g) & \\
\hline
\end{tabular}

Table 1: The different Declare models used during the experiments. The figures of the models used in the experiment can be found by following the link to the tool site.

At the start of the test, students were provided instructions making use of the example used in Section 2, a model which was used as a foundation for models 3-5, but without the additional constraints and activities added. As such, the idea behind hidden dependencies was explained, as well as how to make use of the tool they were provided with.

In order to measure the impact of handing natural language descriptions and the visualization of dependency graphs, the students were divided into three groups which received a different version of the Declare Execution Environment. Group A could only see the Declare model and the constraint descriptions, but no color annotation nor dependency structure visualizations. Group B received a tool in which the enabled activities were colored green, and temporarily violated constraints were colored red, in a fashion described in [10] and similar to Declare Designer [12]. Also, the constraint descriptions were given. Finally, group C was given an environment with the same functionality as group B, but with extra 


\begin{tabular}{|r|r|rr|rrr|}
\hline & & \multicolumn{2}{|c|}{ Gender } & \multicolumn{3}{|c|}{ Program } \\
\hline Group & Participants & \multicolumn{2}{|c|}{ Male Female } & IS & Business & CS \\
\hline A & 36 & 25 & 11 & 5 & 31 & 0 \\
B & 32 & 23 & 9 & 6 & 26 & 0 \\
C & 27 & 15 & 12 & 5 & 21 & 1 \\
\hline
\end{tabular}

Table 2: The students were selected from 3 different programs, however, it was made sure their distribution could not skew the results.

descriptions concerning hidden dependencies, as well as the possibility to open a dynamic visualization of the dependency structures.

The questions were aimed at uncovering to which extent the participants grasped the full impact of the blend of different constraints. They were asked to indicate which activities were enabled after firing a certain sequence, and why or how to reach a certain firing sequence. Since two out of three groups knew which ones were enabled, they could focus more on the second part of the question. An example question used for model 1 is 'After firing D, which activities are still enabled? Explain..

Each question was scored on a 0 to 1 scale, where incomplete answers (usually because of overlooked hidden dependencies or incorrect use of constraints) were still awarded a score higher than 0. E.g., a student from group B who provides the correct set of enabled activities but fails to state that activity $c$ in model 3 is not enabled because of hidden dependencies was still awarded 0.6. The explanation was taken into account so as to make a fair comparison with students in group A, who got no extra information, and therefore many times missed even these basic answers. Group C students that just copied extra descriptions provided by the tool also did no receive a grade of 1 , as they did not prove to understand the model.

\section{$5.2 \quad$ Results}

Quantitative Results Given this setup, an experimental analysis can be conducted to investigate the impact of the environment students were given (i.e. group) on the score with a higher score indicating a better level of understanding. Figure 3 shows boxplots of the average scores over 5 questions, per model and per group. From the figure, it can be seen that for each model, an increase is observed in terms of the score when students are provided with additional hidden dependency-based annotations. Note that the data is available on the tool's web site.

So as to evaluate the statistical significance of this pattern, a linear regression (Score $=\alpha \times$ model $+\beta \times$ group $+\epsilon)$ was fitted on the data. From the results in Table 3 it is clear that both the impact of the model as well as the group (and hence tool) is highly significant. Observe that the data was also fitted for a model with interaction between model and group and also for a model with gender and program included. These models did not raise the R-squared values much $(<0.18)$, hence hinting at little extra explanation power. Running a DurbinWatson-test also rejected the hypothesis for correlation among the residuals. 
Finally, it was tested whether the error terms were distributed normally, as can be seen in Figure 4a.

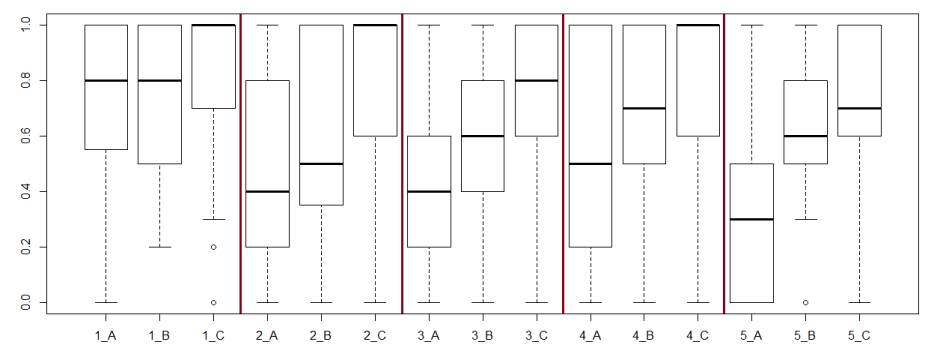

Fig. 3: Boxplot of the average scores of 5 questions per model (1-5) and per group (A-C).

\begin{tabular}{|r|r|r|r|l|r|l|}
\hline Coefficients & Estimate & Std. Error & t value & & $\mathbf{P r}(>|\mathbf{t}|)$ & \\
\hline (Intercept) & 0.64092 & 0.01583 & 40.498 & $<$ & $2.00 \mathrm{E}-16$ & $* * *$ \\
Model2 & -0.17082 & 0.01939 & -8.81 & $<$ & $2.00 \mathrm{E}-16$ & $* * *$ \\
Model3 & -0.17082 & 0.01939 & -8.81 & $<$ & $2.00 \mathrm{E}-16$ & $* * *$ \\
Model4 & -0.10498 & 0.01943 & -5.403 & $<$ & $7.22 \mathrm{E}-08$ & $* * *$ \\
Model5 & -0.21555 & 0.01964 & -10.977 & $<$ & $2.00 \mathrm{E}-16$ & $* * *$ \\
GroupB & 0.15811 & 0.01463 & 10.81 & $<$ & $2.00 \mathrm{E}-16$ & $* * *$ \\
GroupC & 0.26522 & 0.01524 & 17.4 & $<$ & $2.00 \mathrm{E}-16$ & $* * *$ \\
\hline Residual standard error: 0.2986 on 2340 degrees of freedom \\
Multiple R-squared: 0.1658 \\
Adjusted R-squared: 0.1636 \\
F-statistic: 77.49 on 6 and 2340 DF, p-value: < 2.2e-16 \\
\hline
\end{tabular}

Table 3: Linear regression model based on the data gathered from the experiment with significance scores ${ }^{* * * *} 0,{ }^{\prime * *}, 0.001$, and ${ }^{*}, 0.01$.

Qualitative Results Since the participants did not just give an answer in the form of 'A is now enabled' but had to motivate their answers, some extra observations can be made concerning the results. Although it was the case that the two groups with the more elaborate tool were better capable of seeing which activities are enabled and which constraints are violated, they still seemed to ignore these annotations. Especially group B sometimes ignored the coloring of the model as they did not understand some implications of the constraints. Participants often also bended the descriptions of the Declare constraints towards their understanding, hence starting to discuss irrelevant parts of the model. For the third group, this behavior was still present, although to a much lesser extent. Group A participants often found the hidden dependencies in the easier examples, because they had no support they thought harder about the model, but failed to find any in the elaborate examples.

Remarks As in all empirical experiments, there are threats to validity that need to be addressed, the main ones in our case are: 


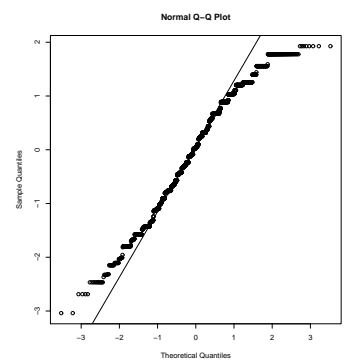

(a) Q-Q plot of the error terms showing they are close to a normal distribution.

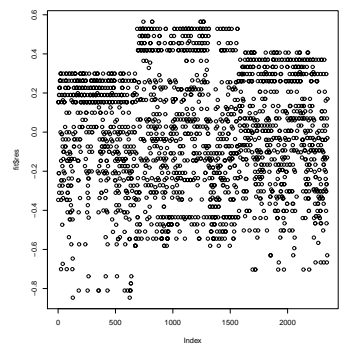

(b) Plot of the residuals, showing no noticeable patterns.

Fig. 4: Descriptive statistics of the results of the linear regression model.

- Internal validity: Our experiment had the maturation threat because subjects may react differently as time passes (because of boredom or fatigue). We solved this threat by dividing the experiment into different questions per model. Also, we made sure there could be no interaction between the students of different sessions.

- Construct validity: Our experiment was threatened by the hypothesis guessing threat because students might figure out what the purpose of the study is, which could affect their guesses. We minimized this threat by hiding the goal of the experiment. Since the R-squared values were not very high, it might also be interesting for further study to include the time spent on the questions and the grades of the final exam of the students to explain the score through the capabilities to learn and understand logic in general.

- External validity: Our experiment might suffer from interaction of selection and treatment: the subject population is limited to students. Although the number of subjects is quite high and their profiles quite balanced, we can only generalize the results to students, but the subjects might not be representative to generalize the results to professional modelers as well. It is, e.g., not possible to claim that the tool can help or improve Declare modeling efforts of more experienced users.

\section{Related Work}

Declare, introduced as DecSerFlow and ConDec in [14]15, has become one of the most widely-used declarative process languages in research. Some competing approaches exist such as DCR Graphs [16], which are comparable to a slimmeddown version of Declare for improving understandability and setup, and the more data-oriented language Guard-Stage Milestone [17.

Declare and its understandability has been researched for a test case-driven approach [4, the impact of hierarchies [1], and its common understandability 
challenges [2]. While these works clearly state the presence of hidden dependencies, with [2] explicitly mentioning this as a common pitfall for understandability, they have not provided a way to capture them. This work continues on the preliminary approach for retrieving hidden dependencies of $[18$.

Many other works on Declare mining exist as well, which have led to a better understanding of the properties of the language. Most notably the hierarchy [8] and semantics 9]19]20] and the transitivity properties 21] have brought clarification as to how constraints behave in a model.

\section{Conclusion and Future Work}

This paper showed how to retrieve and use dependency structures and unary propagation in Declare models to raise understandability. It offers a theoretic aspect in explaining how to construct and interpret the relations of constraints and their hidden dependencies in ways that have not been proposed yet, and was validated on novice users in an experiment. This showed that explaining and visualizing hidden dependencies and constraint structures rendered users significantly better capable of understanding the models.

Future work includes extending these findings to $n$-ary constraints, which changes only the propagation and interpretation slightly. Furthermore, constructing the hidden dependencies has numerous other applications. By understanding in which way constraints are related, it becomes easier to grasp the complexity of multiplying the separate Declare constraints' automata and hence it is possible to score the impact of different constraints on, e.g., the performance of calculating the global automaton of the whole model. Furthermore, these insights can be used to score a Declare model for simplicity. Models which contain more hidden dependencies can be scored lower for this metric, hence offering the currently missing evaluation parameter used in conformance checking frameworks 22 .

\section{References}

1. Zugal, S., Pinggera, J., Weber, B., Mendling, J., Reijers, H.A.: Assessing the impact of hierarchy on model understandability-a cognitive perspective. In: Models in Software Engineering. Springer (2012) 123-133

2. Haisjackl, C., Zugal, S., Soffer, P., Hadar, I., Reichert, M., Pinggera, J., Weber, B.: Making sense of declarative process models: Common strategies and typical pitfalls. In: Enterprise, Business-Process and Information Systems Modeling. Springer (2013) 2-17

3. Pesic, M., Schonenberg, H., van der Aalst, W.M.: Declare: Full support for looselystructured processes. In: Enterprise Distributed Object Computing Conference, 2007. EDOC 2007. 11th IEEE International, IEEE (2007) 287-287

4. Zugal, S., Pinggera, J., Weber, B.: The impact of testcases on the maintainability of declarative process models. In: Enterprise, Business-Process and Information Systems Modeling. Springer (2011) 163-177 
5. Montali, M., Pesic, M., Van Der Aalst, W.M., Chesani, F., Mello, P., Storari, S.: Declarative specification and verification of service choreographiess. ACM Transactions on the Web (TWEB) 4(1) (2010) 3

6. De Smedt, J., De Weerdt, J., Vanthienen, J.: Fusion miner: Process discovery for mixed-paradigm models. Decision Support Systems 77 (2015) 123-136

7. Pesic, M.: Constraint-based workflow management systems: shifting control to users. PhD thesis, Technische Universiteit Eindhoven (2008)

8. Di Ciccio, C., Mecella, M.: A two-step fast algorithm for the automated discovery of declarative workflows. In: Computational Intelligence and Data Mining (CIDM), 2013 IEEE Symposium on, IEEE (2013) 135-142

9. Westergaard, M., Stahl, C., Reijers, H.A.: Unconstrainedminer: Efficient discovery of generalized declarative process models. Technical Report BPM-13-28, BPMcenter (2013)

10. Maggi, F.M., Montali, M., Westergaard, M., Van Der Aalst, W.M.: Monitoring business constraints with linear temporal logic: An approach based on colored automata. In: Business Process Management. Springer (2011) 132-147

11. De Smedt, J., De Weerdt, J., Vanthienen, J., Poels, G.: Mixed-paradigm process modeling with intertwined state spaces. Business \& Information Systems Engineering (2016)

12. Westergaard, M., Maggi, F.M.: Declare: A tool suite for declarative workflow modeling and enactment. BPM (Demos) 820 (2011)

13. Møller, A.: dk. brics. automaton-finite-state automata and regular expressions for java, 2010

14. van der Aalst, W.M.P., Pesic, M.: Decserflow: Towards a truly declarative service flow language. In: The Role of Business Processes in Service Oriented Architectures, 16.07. - 21.07.2006. (2006)

15. Pesic, M., van der Aalst, W.M.: A declarative approach for flexible business processes management. In: Business Process Management Workshops, Springer (2006) $169-180$

16. Hildebrandt, T.T., Mukkamala, R.R.: Declarative event-based workflow as distributed dynamic condition response graphs. arXiv preprint arXiv:1110.4161 (2011)

17. Hull, R., Damaggio, E., Fournier, F., Gupta, M., Heath III, F.T., Hobson, S., Linehan, M., Maradugu, S., Nigam, A., Sukaviriya, P., et al.: Introducing the guard-stage-milestone approach for specifying business entity lifecycles. In: Web services and formal methods. Springer (2011) 1-24

18. De Smedt, J., De Weerdt, J., Serral Asensio, E., Vanthienen, J.: Gamification of declarative process models for learning and model verification. In: Business Process Management Workshops, Springer (2015)

19. De Giacomo, G., Masellis, R.D., Montali, M.: Reasoning on LTL on finite traces: Insensitivity to infiniteness. In: Proceedings of the Twenty-Eighth AAAI Conference on Artificial Intelligence, July 27 -31, 2014, Québec City, Québec, Canada. (2014) 1027-1033

20. Di Ciccio, C., Mecella, M., Mendling, J.: The effect of noise on mined declarative constraints. In: Data-Driven Process Discovery and Analysis. Springer (2015) 1-24

21. Maggi, F.M., Bose, R.J.C., van der Aalst, W.M.: A knowledge-based integrated approach for discovering and repairing declare maps. In: Advanced Information Systems Engineering, Springer (2013) 433-448

22. de Leoni, M., Maggi, F.M., van der Aalst, W.M.: An alignment-based framework to check the conformance of declarative process models and to preprocess event-log data. Information Systems 47 (2015) 258-277 